\title{
LA-UR-16-22490
}

Approved for public release; distribution is unlimited.

Title: $\quad$ Safeguards Technology Factsheet Unattended Dual Current Monitor (UDCM)

Author(s): $\quad$ Newell, Matthew R.

Intended for: Report

Issued: 
Disclaimer:

Los Alamos National Laboratory, an affirmative action/equal opportunity employer,is operated by the Los Alamos National Security, LLC for the National NuclearSecurity Administration of the U.S. Department of Energy under contract DE-AC52-06NA25396. By approving this article, the publisher recognizes that the U.S. Government retains nonexclusive, royalty-free license to publish or reproduce the published form of this contribution, or to allow others to do so, for U.S. Government purposes. Los Alamos National Laboratory requests that the publisher identify this article as work performed under the auspices of the U.S. Departmentof Energy. Los Alamos National Laboratory strongly supports academic freedom and a researcher's right to publish; as an institution, however, the Laboratory does not endorse the viewpoint of a publication or guarantee its technical correctness. 


\title{
Safeguards Technology Factsheet
}

\section{Unattended Dual Current Monitor (UDCM)}

\author{
LA-UR-16-????
}

\section{Summary}

The UDCM is a low current measurement device designed to record sub-nano-amp to micro-amp currents from radiation detectors. The UDCM is a two channel device that incorporates a Commercial-Off-The-Shelf (COTS) processor enabling both serial over USB as well as Ethernet communications. The instrument includes microSD and USB flash memory for data storage as well as a programmable High Voltage (HV) power supply for detector bias. The UDCM is packaged in the same enclosure, employs the same processor and has a similar user interface as the UMSR. A serial over USB communication line to the UDCM allows the use of existing versions of MIC software while the Ethernet port is compatible with the new IAEA RAINSTORM communication protocol.

\section{Background}

The IAEA is in need of a simple and inexpensive data acquisition system for unattended current monitoring when using detectors like ion chambers. The system currently approved for unattended use, the MiniGRAND, is no longer available and the recently designed UNAP system, although a great solution for complex systems, is not appropriate for a majority of the IAEA needs. LANL recently developed two current monitoring devices for this application, 1) the replacement dual current board for the UNAP instrument and 2) the Current to Pulse Converter (CPC). Both of these devices are based on the proven and sound Dual Current Mode GRAND III transimpedance amplifier circuit. However the cost, complexity and size of the UNAP and the lack of Ethernet connectivity of the CPC make these devices less than ideal for unattended operation. The UDCM combines the proven transimpedance amplifier with a simple commercial off the shelf processor board resulting in a low cost, simple, small unattended replacement for the MiniGRAND current monitor.
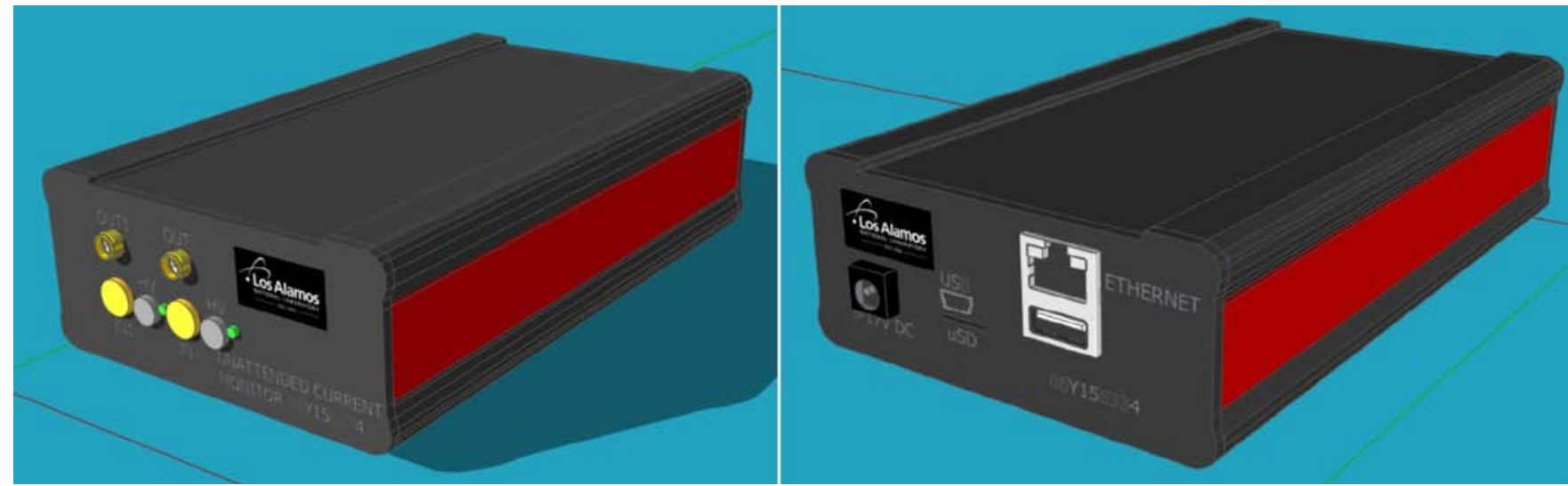

Figure 1. Conceptual Drawing of UDCM. 


\section{Los Alamos}

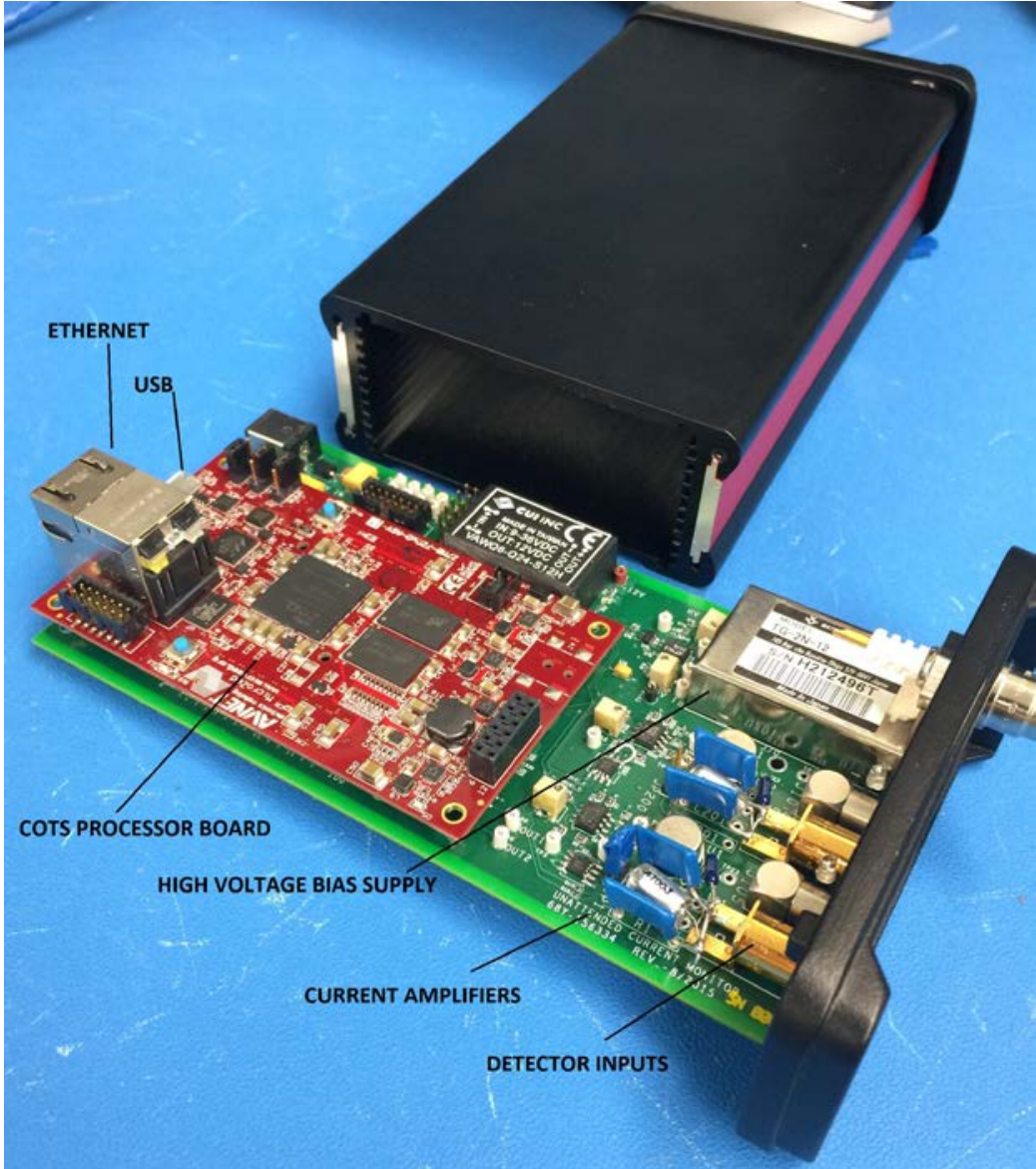

Figure 2. Picture of disassembled prototype UDCM.

\section{Func tional Elements}

- Weight/Dimensions: < 800g / 180x100x50 $\mathrm{mm}^{3}$.

- Power requirements: 9-17V, 3W.

- Inputs: Two Channel low current inputs from -0.2nA to -2uA.

- Outputs: 1) Negative high voltage detector bias (0 to $-1 \mathrm{kV})$,

2) Two Channel TTL output frequency proportional to input current.

- Communications: Ethernet (RAINSTORM compatible), Serial over USB (MIC compatible), USB2.0 host.

- Temperature: -10 to 50 degrees $\mathrm{C}$.

- Memory: 1GB DDR3 SDRAM, 128Mb QSPI Flash, Micro SD card, USB flash drive. 


\section{Operational Description}

The UDCM is a benchtop instrument that is connected to low current producing radiation detectors via coaxial cables. Detector currents from $-0.2 \mathrm{nA}$ to $-2.0 \mathrm{uA}$ can be measured with two simple gain settings. HV bias is available from the front panel of the UDCM using coaxial connectors identical to the connectors of legacy instruments. The operator can connect a laptop running MIC software directly to the UDCM using a standard USB cable and operate in serial communications mode. Alternatively the UDCM can be connected to a Local Area Network (LAN) enabling the user to connect to the UDCM over the local network.

Configuring the UDCM is accomplished over the communication ports described above and using MIC or equivalent software. The UDCM configurable parameters are: high or low gain setting, HV bias value, and measurement time. Data can be stored on the user accessible micro SD card, a USB flash drive or downloaded directly to the user's computer.

\section{FY16 Status}

The UDCM is currently under development. The first prototype, shown in Figure 2, has been built and is now being tested. Basic functions like reporting measured current to a web browser and production of pulse streams proportional to the input current are implemented. A user's manual as well as a test results document will be produced.

\section{References}

[1] R. Parker, S. Klosterbuer, “MiniGRAND User Manual," Los Alamos National Laboratory report LA-UR-994400 (December 1999). 